\title{
Pemphigus foliaceus with pustular presentation in a patient with psoriasis*
}

\author{
Vando Barbosa de Sousa ${ }^{1}$ \\ Daniele do Nascimento Pereira ${ }^{1}$
}

\author{
Cândida Naira Lima e Lima Santana ${ }^{1}$ \\ Alexandre Carlos Gripp ${ }^{1}$
}

DOI: http:/ /dx.doi.org/10.1590/abd1806-4841.20175709

\begin{abstract}
Pemphigus foliaceus is a chronic autoimmune disease of the skin, clinically characterized by scaly and crusty cutaneous erosions involving the seborrheic areas. The patient can eventually become erythrodermic. There are reports of atypical cases of pemphigus foliaceus with pustules and neutrophils, and clinical differentiation from generalized pustular psoriasis of von Zumbusch is difficult. We report the case of a 55-year-old man with a history of psoriasis vulgaris that has developed pemphigus foliaceus with pustules, triggered by withdrawal of systemic corticosteroids. This is the first report associating this atypical form of pemphigus with psoriasis, suggesting that an overlap with generalized pustular psoriasis can occur.
\end{abstract}

Keywords: Vesiculobullous conditions; Pemphigus; Psoriasis

\section{INTRODUCTION}

Pemphigus foliaceus (PF) is a chronic autoimmune skin condition characterized by subcorneal acantholytic bullae and IgG1 and IgG4 antibody deposition in the keratinocyte transmembrane proteins (desmoglein-1 and -3). Generalized pustular psoriasis of von Zumbusch (GPP) is an uncommon variant of psoriasis, that presents sterile pustules on erythematous skin, systemic signs and symptoms and laboratory abnormalities similar to neutrophilic diseases. It can be triggered by intrinsic and extrinsic factors, being the most common systemic corticosteroid withdrawal. Atypical PF cases that present with neutrophilic pustules have been described, making the differential diagnosis with GPP difficult. ${ }^{1-3}$ However, a relationship between both conditions has not yet been established. The association between psoriasis and PF is rare, 11 cases having been described in the literature in English. Among those, almost all were of psoriasis vulgaris. ${ }^{4-8}$

\section{CASE REPORT}

A 55-year-old male patient with psoriasis vulgaris since he was 20 years old had been stable for a long time with the use of topical steroids; he had scaly plaques on the scalp, elbows and hands. The clinical picture evolved with the appearance of new erythematous, scaly lesions on the back, when PF was considered and later confirmed by skin biopsy and direct immunofluorescence (DIF). Prednisone (40 mg/day), was prescribed, with partial improvement. Prednisone was slowly tapered and, upon reaching the dose of $5 \mathrm{mg} /$ day, he had a sudden worsening, with an erythematous scaly eruption affecting the whole trunk. Thus, the prednisone dose was increased ( $80 \mathrm{mg} /$ day), but there was no improvement and he developed pustular lesions and fever spikes (Figure 1). He sought our department, and we reviewed his previous histopathology, that showed changes suggestive of psoriasis. New biopsies with samples for DIF were performed, that confirmed the diagnosis of PF (Figure2). While waiting for the histopathology and DIF results, we started

Work submitted on 17.02.2016

Approved by the Advisory Board and accepted for publication on 12.08.2016

* Study conducted at Hospital Universitário Pedro Ernesto, da Universidade do Estado do Rio de Janeiro (Hupe-Uerj) - Rio de Janeiro (RJ), Brazil. Financial Support: None.

Conflict of Interests: None.

1 Dermatology Department at Hospital Universitário Pedro Ernesto, of the Universidade do Estado do Rio de Janeiro (Hupe-Uerj) - Rio de Janeiro (RJ), Brazil. 

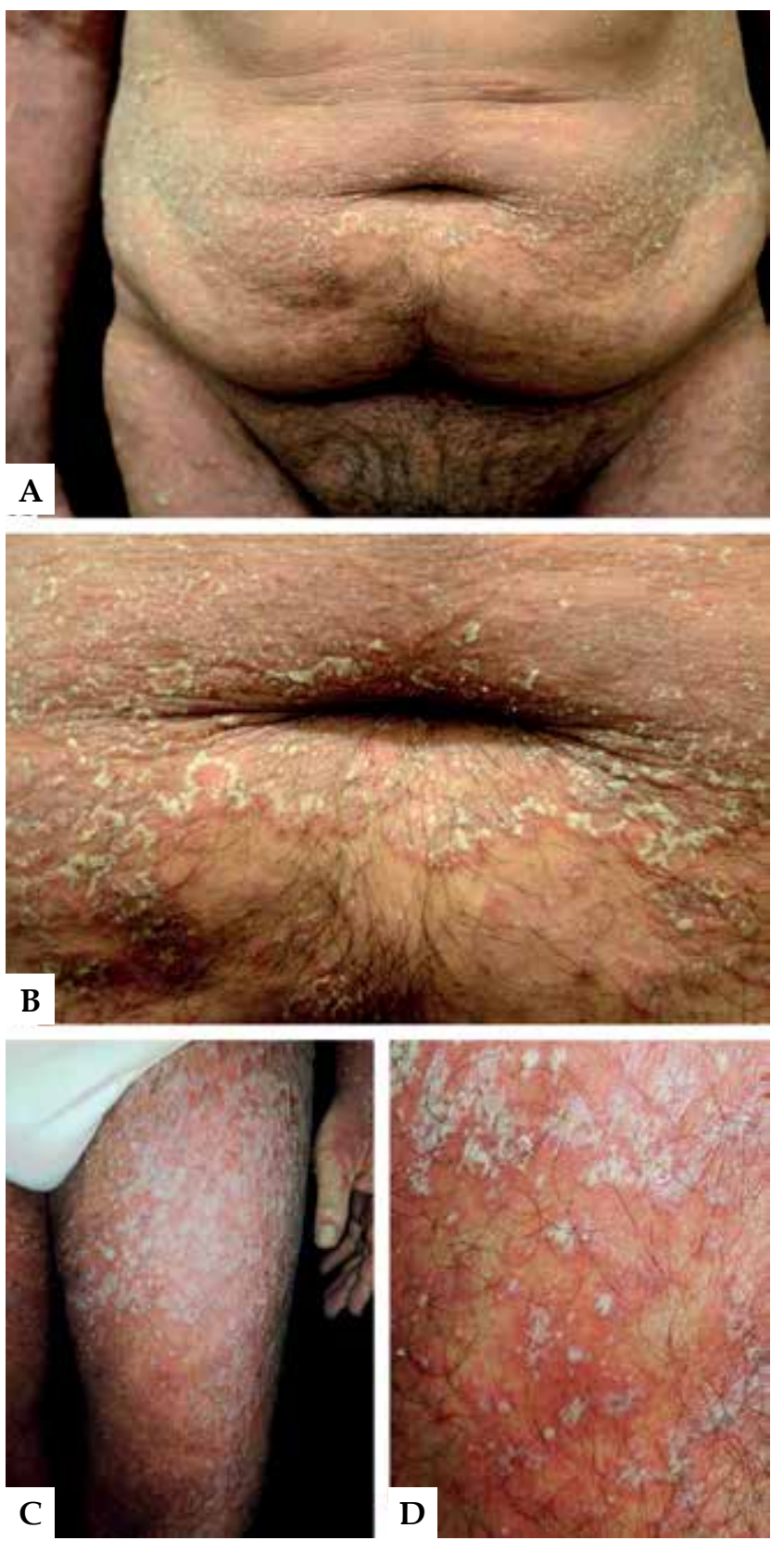

Figure 1: (A) Erythema and scaling on the abdominal region.

(B) Peripheral pustules. (C) and (D) Clinical worsening after corticosteroid tapering: confluent pustules over an erythematous base

cyclosporine and acitretin. With the test results, we added immunoglobulin and he eventually improved. During follow-up, he had a complicated acute diverticulitis and underwent a surgical procedure with placement of colostomy. We continued to taper the corticosteroid slowly, but he had a new flare of the pustular lesions when prednisone dose was lowered to $10 \mathrm{mg} /$ day (Figure 1). The patient is currently under follow-up in our department.

\section{DISCUSSION}

$\mathrm{PF}$ is a chronic autoimmune skin condition characterized by IgG antibody formation against desmoglein-1 and, rarely, desmoglein-3 - these are the proteins that form the desmosomes, adhesion structures between keratinocytes - , resulting in the formation of intraepidermal blisters. Clinically, it presents as scaly, crusty and eroded lesions over an erythematous base, affecting seborrheic areas. The blisters might not be obvious because they are formed superficially in the epidermis and rupture quickly. There can eventually be progression to erythroderma.

GPP is an uncommon variant of psoriasis, with the eruption of sterile pustules and systemic symptoms. The patient can present with any pustular variant: isolated pustules, collections of pus, circinate lesions, erythematous plaques with a pustular border and generalized erythroderma. There is substantial evidence that therapeutic oral corticosteroid withdrawal can trigger GPP. Other triggers include infections, pregnancy, hypocalcemia, topical and systemic medications. ${ }^{9}$

The association between psoriasis and PF is not explained in the literature. Considering the few cases described of the association of both conditions, in addition to the high prevalence of psoriasis in the population, a causal relationship is possible. However, the similarities between both conditions suggest that the simultaneous occurrence might not be coincidental. ${ }^{8}$ HLA genotype overlap and abnormal activation of T-cells can cause psoriasis and PF. Besides, the chronic stimulation of the immune system that leads to the phenomenon of epitope spreading can be the background for the development of PF after years of untreated psoriasis. ${ }^{5}$ There are other related triggers still, such as narrow band-UVB irradiation, leading to an inflammatory process and exposure of desmoglein in the epidermis. ${ }^{4}$

The onset of a typical pustular eruption in a psoriasis patient during tapering of systemic corticosteroids lead us to strongly consider the diagnosis of GPP. However, our case surprised us with histologic features consistent with the diagnosis of PF. DIF is indispensable for the diagnosis of $\mathrm{PF}$, demonstrating the deposition of IgG antibodies between keratinocytes.

It must be taken into account: 1) PF can rarely manifest with neutrophilic pustules, making the differential diagnosis with GPP difficult; ${ }^{1-3}$ and 2) the diagnosis of GPP can not be ruled out, given the history of psoriasis and the clinical progression. So far, there have been no reports of the simultaneous occurrence of PF and GPP, but the findings direct us to consider that an overlap might be possible.

The reasons for the appearance of pustules in PF are not clear. Non-classical presentations of pemphigus could be explained by the difference in the epitopes that react against antidesmoglein-1. This could explain the difference between the phenotypes of PF and pemphigus herpetiformis, for example. ${ }^{3}$ Besides, IL-8 expression has been associated with neutrophil recruitment. ${ }^{1-3}$ A significant increase in the levels of IL-8 has also been shown in psoriasis. ${ }^{10}$

A pustular eruption in the context of PF is a rare occurrence and its mechanism is still uncertain. Previous reports have not identified a history of psoriasis, despite the fact that the reported cases 

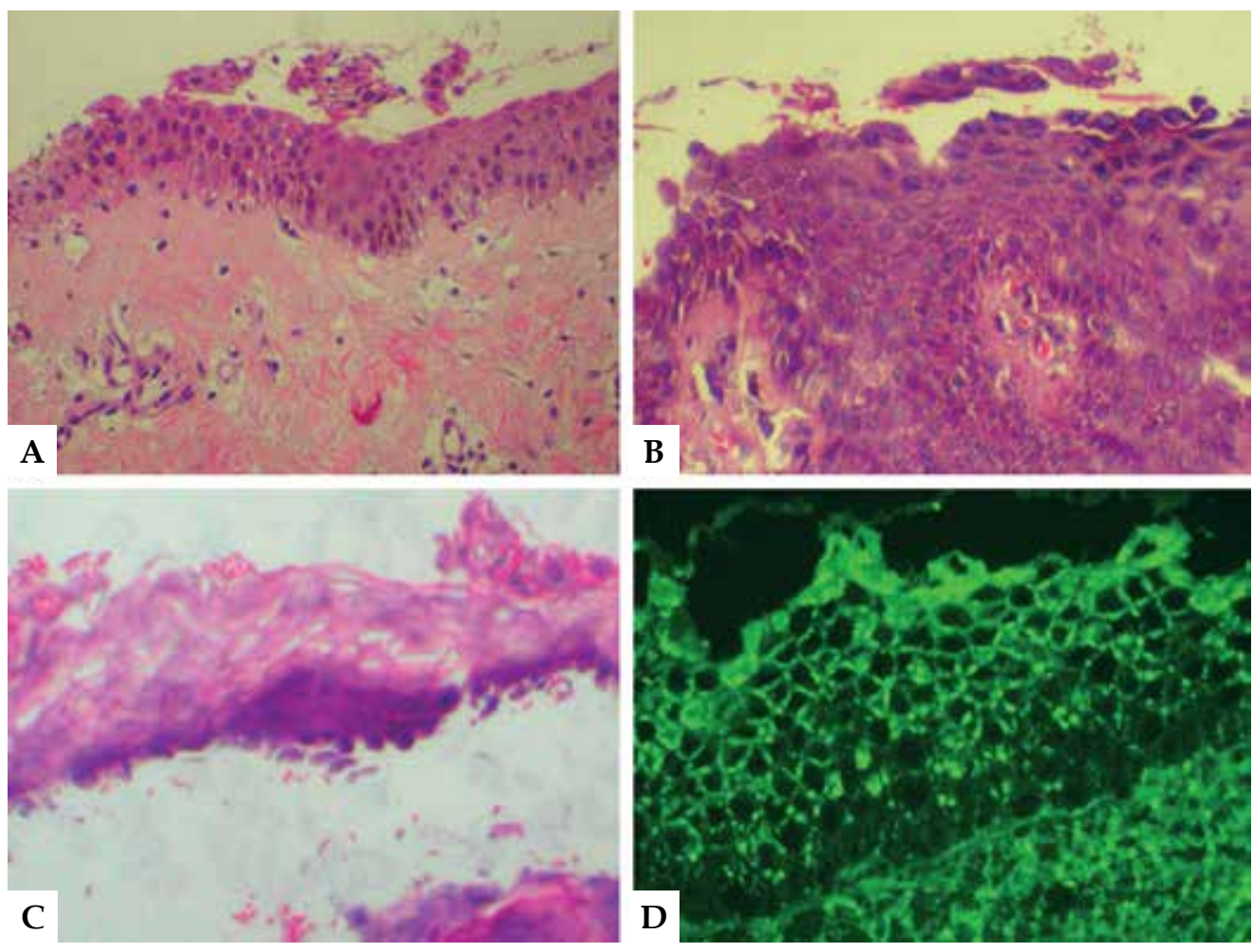

Figure 2: A, B and C: Extensive acantholysis in the upper layers of the epidermis (Hematoxylin \& eosin; A: X100, B: X100 and C: X400). D: Direct immunofluorescence demonstrating the deposition of intrapidermal, intercellular IgG (X400)

simulated GPP clinically. ${ }^{1-3}$ This is the first case where there is an association with psoriasis, suggesting that GPP might be implicated in the origin of pustular lesions of PF. $\square$

\section{AKNOWLEDGEMENTS}

The authors would like to thank the collaboration of the professors Juan Manuel Pineiro-Maceira and Aline Lopes Bressan.

9. Griffiths CEM, Barker JNWN. Psoriasis. In: Burns T Burns, S. Breathnach, N. Cox and C. Griffiths, editors. Rook's Textbook of dermatology 8th ed. Oxford: WileyBlackwell; 2010. p.20.48-9

10. Coimbra S, Oliveira H, Reis F, Belo L, Rocha S, Quintanilha A, et al. Interleukin (IL)-22, IL-17, IL-23, IL-8, vascular endothelial growth factor and tumour necrosis factor- $\alpha$ levels in patients with psoriasis before, during and after psoralen-ultraviolet A and narrowband ultraviolet B therapy. Br J Dermatol. 2010;163:1282-90.

How to cite this article: Sousa VB, Lima-Santana CNL, Pereira DN, Gripp AC. Pemphigus foliaceus with pustular presentation in a patient with psoriasis. An Bras Dermatol. 2017;92(5 Suppl 1): 115-7. 